\title{
Aeroelastic Wing Shaping Control Subject to Actuation Constraints
}

\author{
Sean Shan-Min Swei*, Nhan Nguyen ${ }^{\dagger}$
}

\begin{abstract}
This paper considers the control of coupled aeroelastic aircraft model which is configured with Variable Camber Continuous Trailing Edge Flap (VCCTEF) system. The relative deflection between two adjacent flaps is constrained and this actuation constraint is accounted for when designing an effective control law for suppressing the wing vibration. A simple tuned-mass damper mechanism with two attached masses is used as an example to demonstrate the effectiveness of vibration suppression with confined motion of tuned masses. In this paper, a dynamic inversion based pseudo-control hedging (PCH) and bounded control approach is investigated, and for illustration, it is applied to the NASA Generic Transport Model (GTM) configured with VCCTEF system.
\end{abstract}

\section{Introduction}

An earlier research study conducted by NASA has proven that active control of wing aeroelasticity inflight can be effective in reducing aerodynamic induced drag and enhancing lift performance. The analyses showed that through active control of wing twist and deflection at local wing sections the overall aerodynamic efficiency can be improved. As a result, the novel concept of Variable Camber Continuous Trailing Edge Flap system is proposed. ${ }^{1}$ Two sets of control actuators are employed in order to actuate the VCCTEF. The light-weight shaped memory alloy (SMA) is adapted for controlling the shape of the first two chordwise sections of the three-section VCCTE flap. The thrid section is controlled by the electric drive motor (EDM) and provides the needed active wing shaping control in-flight. A preliminary static analysis has shown the potential efficacy of the VCCTEF system.

This paper presents the mechanical modeling of the VCCTEF system and incorporates it into aeroelastic aircraft dynamics. A single degree-of-freedom vibration with tuned-mass damper (TMD) of two attached masses is considered. The main mass-dashpot-spring setup simulates the one degree-of-freedom vibration of the flexible wing, for example, first bending mode. The attached TMDs are confined in their relative motion and they simulate the two adjacent VCCTEFs. The TMD is well understood in the mechanical and civil engineering community as a device used for vibration absorber. ${ }^{2,3}$ However, the TMD with confined motion is a novel concept.

The actuation of VCCTEFs is both bounded and constrained by deflection angle and deflection rate. This is due to the placement of elastomer material in between the flaps to provide piecewise continuous motion. However, the presence of elastomer further complicates the control system design, since it renders confined motion between the two flaps. Previous works on bounded input and input rate, for instance ${ }^{6-8}$ and the references therein, offer a good framework for control designers to embark on the implementation aspect of the proposed approaches. A simple "software limiter" idea was proposed in Hess and Snell. ${ }^{7}$ There, by introducing the derivative and limited integration blocks a commanded input signal can be re-generated. In $\operatorname{Lin}^{8}$ a bounded input and input rate problem description was given that, in a sense, prescribes how the actuator should be moving. In this paper, we will utilize the bounded control approach proposed in. ${ }^{6}$ A higher order state-space system was obtained by augmenting the states with the control, hence, in this setup the new control to the augmented system is in fact the input rate. Inevitably, the control structure in this case becomes a dynamic controller.

The pseudo-control hedging or $\mathrm{PCH}^{5,9}$ is another viable alternative control approach which is well suited for dealing with such practical control issue as actuator saturation. Since a dynamic inversion of the plant

\footnotetext{
*Research Scientist, Intelligent Systems Division, NASA Ames Research Center

${ }^{\dagger}$ Research Scientist, Intelligent Systems Division, NASA Ames Research Center
} 
model is involved, its application is mainly for the stable systems. However, in the context of VCCTEF actuation, we propose to utilize PCH modeling technique to VCCTEF model to achieve better wing shaping control. In this setup, the PCH based VCCTEF control can be regarded as an inner loop design that incorporates any mismatch from the desired control deflection and deflection rate, whereas the bounded input and input rate control can be considered as an outer loop design. This proposed approach is applied to the NASA Generic Transport Model (GTM) configured with VCCTEF system for illustration.

\section{Aeroelastic Aircraft Dynamics with VCCTEF}

Figure 1 shows the GTM with configured VCCTEF system. As shown, there are 11 flaps per wing and they are equally spaced, and each flap has three sections, as shown in Figure 2. Sections 1 and 2 are driven by shape memory alloy (SMA) and produce high lift, whereas section 3 is driven by high bandwidth electric drive motor (EDM) necessary to provide active wing shaping control. At nominal cruise flight, we consider only the section 3 is being activated.

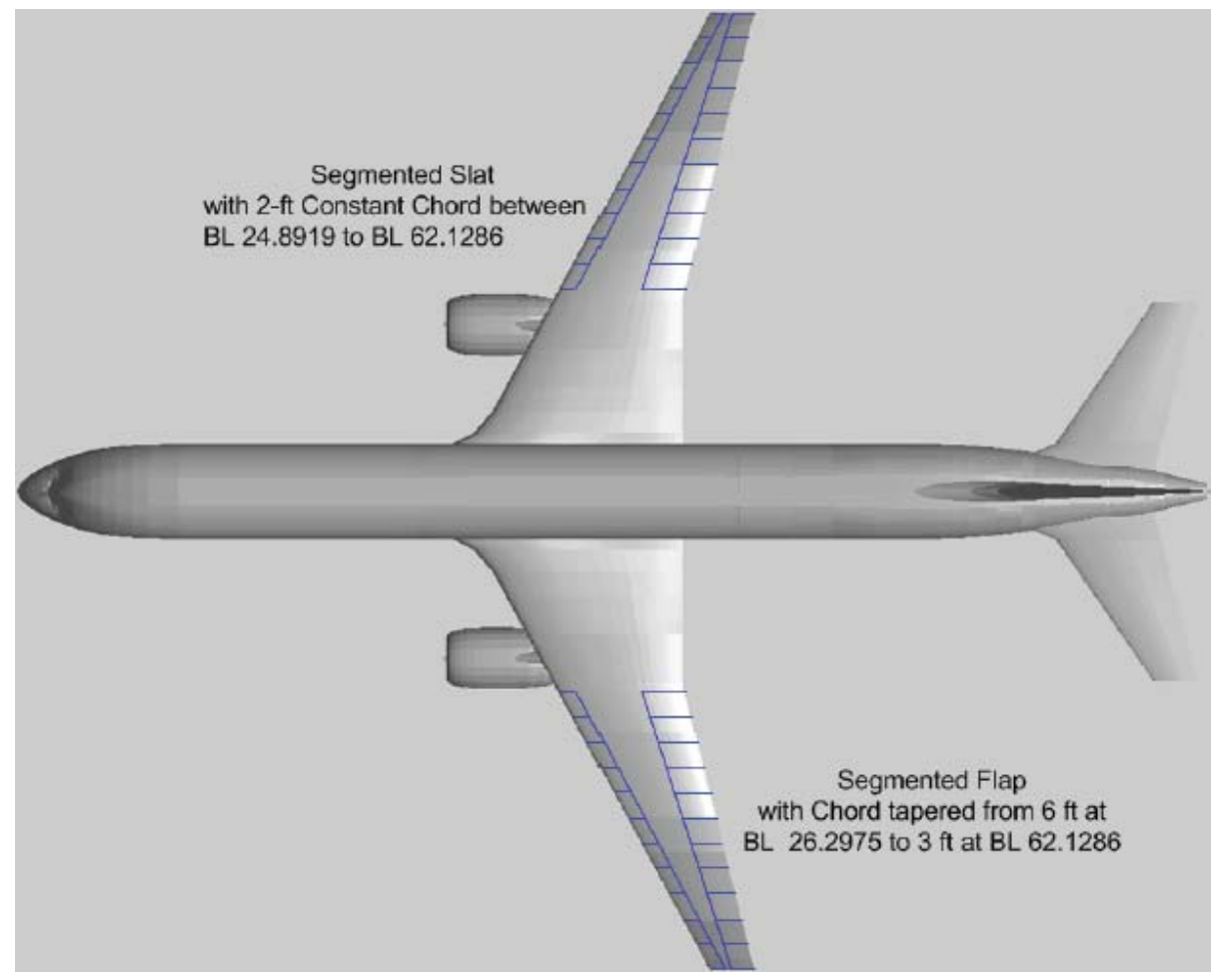

Figure 1. GTM with equally spaced VCCTEF

The equations of motion for coupled aircraft rigid-body dynamics with flexible aeroelastic wing modes at nominal cruise flight can be described by

$$
\underbrace{\left[\begin{array}{c}
\dot{x}_{a} \\
\dot{x}_{e}
\end{array}\right]}_{\dot{x}_{p}}=\underbrace{\left[\begin{array}{cc}
A_{a a} & A_{a e} \\
A_{e a} & A_{e e}
\end{array}\right]}_{A_{p}} \underbrace{\left[\begin{array}{c}
x_{a} \\
x_{e}
\end{array}\right]}_{x_{p}}+\underbrace{\left[\begin{array}{c}
B_{a a} \\
B_{e a}
\end{array}\right]}_{B_{p a}} \delta_{a}+\underbrace{\left[\begin{array}{c}
B_{a e} \\
B_{e e}
\end{array}\right]}_{B_{p e}} \delta_{f}
$$

where $x_{a}$ denotes the aircraft's rigid-body states, $x_{e}$ consists of displacement and velocity of aeroelastic wing at generalized coordinates, ${ }^{4} \delta_{a}$ denotes the control surface deflection, such as elevator, and $\delta_{f}$ denotes the VCCTEF 3rd segment deflection. The matrices $A_{a a}$ and $A_{e e}$ contain aircraft rigid-body and aeroelastic characteristics, whereas $A_{a e}$ and $A_{e a}$ correspond to aeroelastic coupling and aircraft rigid-body coupling, respectively. Similarly, $B_{a e}$ and $B_{e a}$ represent coupling effect between the control surface and VCCTEF. Note that the dimension of overall system depends on the number of rigid-body states and number of aeroelastic modes included in the problem setup. 


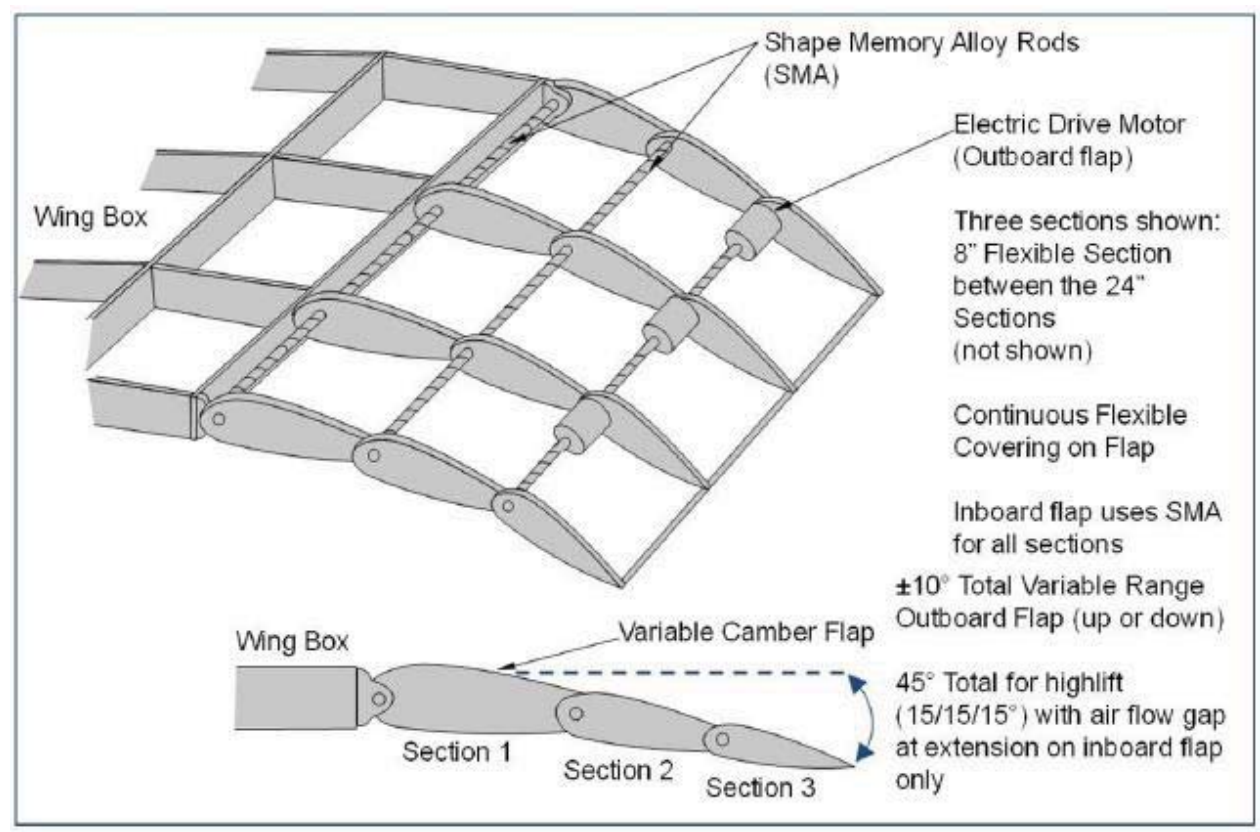

Figure 2. VCCTEF actuation using SMA and EDM

In order to achieve active wing shaping control for drag reduction in flight, it should be noted that each flap can not be operated independently. Therefore, to ensure and approximate smooth and continuous variation of wing shape, it is necessary to confine the relative motion between the two neighboring flaps. To achieve this, an elastomer material is placed between two adjacent flaps, which produces continuous transition for relative motion. However, presence of elastomer also introduces additional constraints on the performance of VCCTEF, and these constraints need to be addressed and incorporated in the control systems design process. The desired relative motion is often prescribed apriori and can be used for control analysis and synthesis.

\section{Integrating Actuator Dynamics with VCCTEF}

Figure 3 shows the schematics of VCCTEF with the elastomer modeled as a pair of spring-dashpot mechanism. As shown, the relative deflection between, for example, $\theta_{1}$ and $\theta_{2}$ is constrained, as well as the relative rate between $\dot{\theta}_{1}$ and $\dot{\theta}_{2}$. It is important to note that the elastomer material properties depend on the operation ambient conditions. In this study, we shall use notional material properties and consider them as potential design parameters for further analysis.

We assume that the EDM actuator is modeled as a second order dynamics of the form

$$
\ddot{\theta}_{i}+2 \zeta \omega_{n} \dot{\theta}_{i}+\omega_{n}^{2} \theta_{i}=\omega_{n}^{2} \delta_{i} ; i=1,2, \cdots, m,
$$

where $\theta_{i}$ and $\dot{\theta}_{i}$ are output of $i$ th actuator and represent respectively the deflection angle and rate of $i$ th flap, and $\delta_{i}$ denotes the control command to the $i$ th flap. The parameters $\zeta$ and $\omega_{n}$ are known damping ratio and natural frequency provided by hardware specifications. When all $m$ flaps are interconnected with 


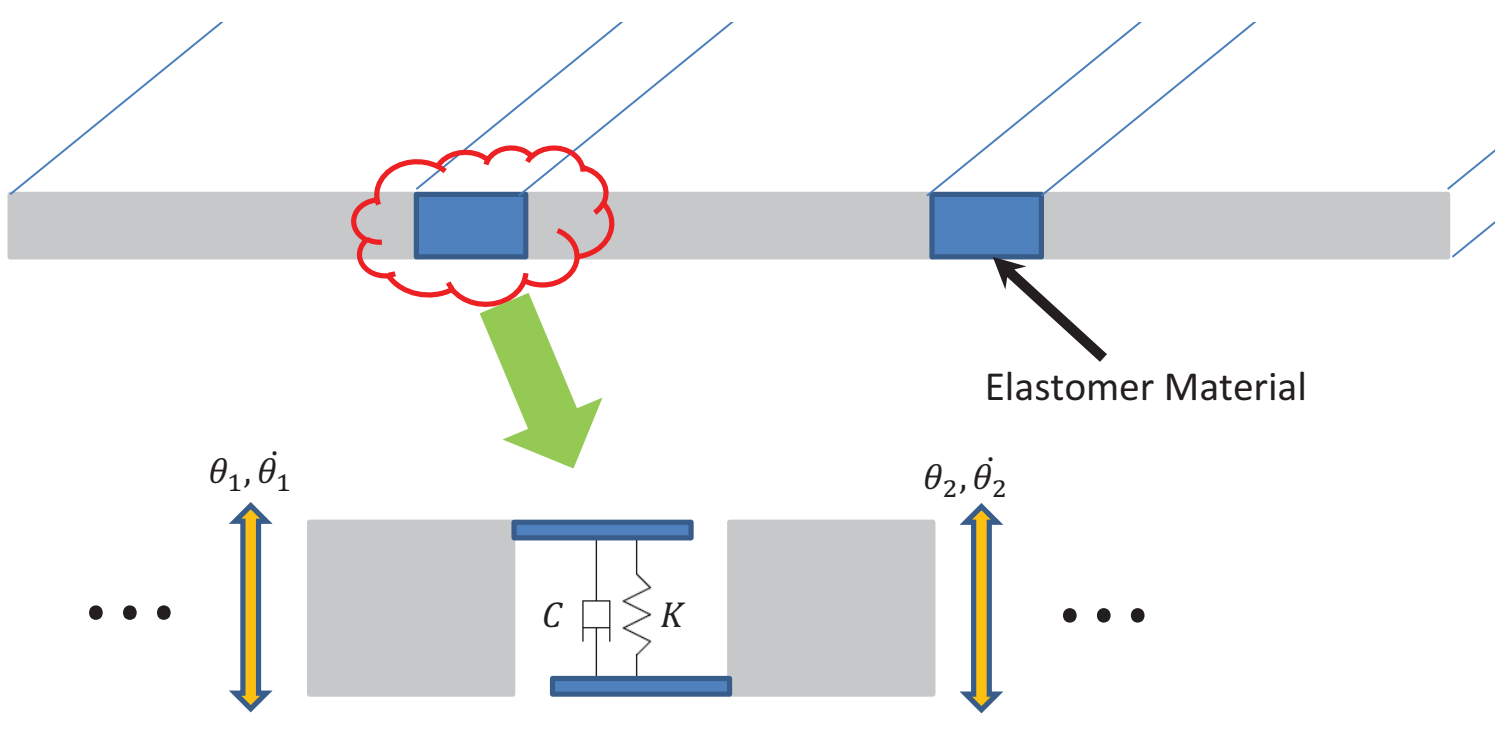

Figure 3. Schematics of VCCTEF

EDM actuators, as shown in Figure 4, the overall actuator dynamics can be described by

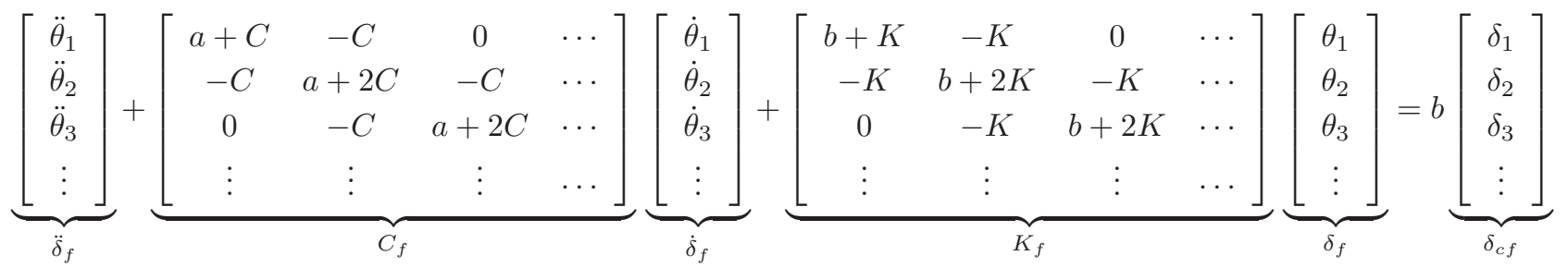

where $a=2 \zeta \omega_{n}, b=\omega_{n}^{2}$, and $\delta_{c f}$ denotes the control command to VCCTEFs. In attaining the above we assumed that all EDM actuator dynamics and elastomer material properties are the same. As noted earlier the relative deflection output from two adjacent flaps is constrained by $\alpha_{i}$, i.e. $\left|\theta_{i-1}-\theta_{i}\right| \leq \alpha_{i}$ and $\left|\theta_{m}\right| \leq \alpha_{m}$. In practical application, the motion of VCCTEF is confined not only by relative angular deflection, and because of bandwidth limitation of actuator dynamics and the presence of elastomer material, the relative angular deflection rate between two adjacent flaps is also bounded, i.e. $\left|\dot{\theta}_{i-1}-\dot{\theta}_{i}\right| \leq \rho_{i}$ and $\left|\dot{\theta}_{m}\right| \leq \rho_{m}$, and both $\alpha_{i}$ and $\rho_{i}$ are known constants.

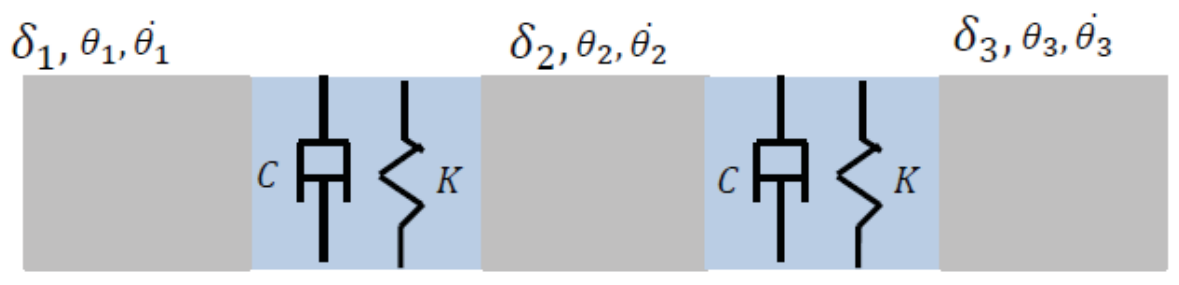

Figure 4. EDM driven VCCTEF $(2)$,

We also assume that the actuator dynamics for control surfaces $\delta_{a}$ takes a second order form similar to

$$
\ddot{\delta}_{a}+C_{a} \dot{\delta}_{a}+K_{a} \delta_{a}=b \delta_{c a}
$$

where $\delta_{a}$ is the control surface deflection angle and $\delta_{c a}$ the control command. As noted earlier, both $\delta_{a}$ and $\dot{\delta}_{a}$ are bounded by motor dynamics.

Now, combining the aeroelastic aircraft dynamics (1) with actuator dynamics (3) and (4) yields the 
following open-loop system description,

$$
\left\{\begin{array}{l}
\dot{x}_{p}=A_{p} x_{p}+B_{p a} \delta_{a}+B_{p e} \delta_{f} \\
\ddot{\delta}_{f}+C_{f} \dot{\delta}_{f}+K_{f} \delta_{f}=b \delta_{c f} \\
\ddot{\delta}_{a}+C_{a} \dot{\delta}_{a}+K_{a} \delta_{a}=b \delta_{c a} \\
y_{p}=C_{p} x_{p}
\end{array}\right.
$$

where $y_{p}$ denotes the control output variables. Figure 5 illustrates the schematic of open-loop block diagram, which shows the aeroelastic aircraft model interconnecting with constrained VCCTEF dynamics.

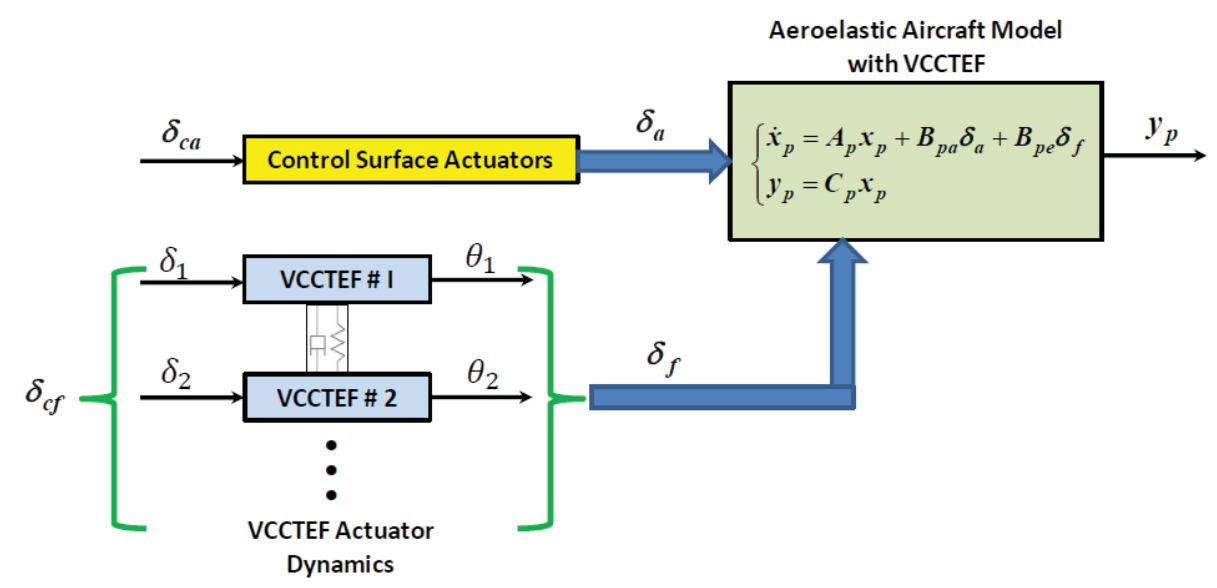

Figure 5. Interconnection of aeroelastic aircraft model with VCCTEF actuator dynamics

\section{A Tuned-Mass Damper Analogy}

In order to demenstrate the efficiency of VCCTEF in suppressing the virbrational motion of the wing, a simplified tuned mass damper (TMD) problem is considered; see Figure 6. As shown, the main massdashport-spring suite $\left(M, c_{1}, k_{1}\right)$ models one vibrational mode, for instance wing's first bending mode, and it is subjected to external excitation force $f(t)$. The attached series of two small masses $m$ are considered as a pair of VCCTEFs. The two dashport-spring sets $\left(c_{2}, k_{2}\right)$ and $\left(c_{3}, k_{3}\right)$ that connect the three masses represent the EDM dynamics and the effect of elastomers. It should be noted that, unlike the conventional tuned mass damper study where displacement of mass $m$ is not constrained, here the relative displacement between $x_{2}$ and $x_{3}$ is confined, that is $\left|x_{2}-x_{3}\right|$ is bounded. The application of tuned mass damper as a tuned vibration absorber is well studied and understood, however, in this study our purpose is to show the effectiveness of the tuned mass damper by confining relative motion between $x_{2}$ and $x_{3}$.

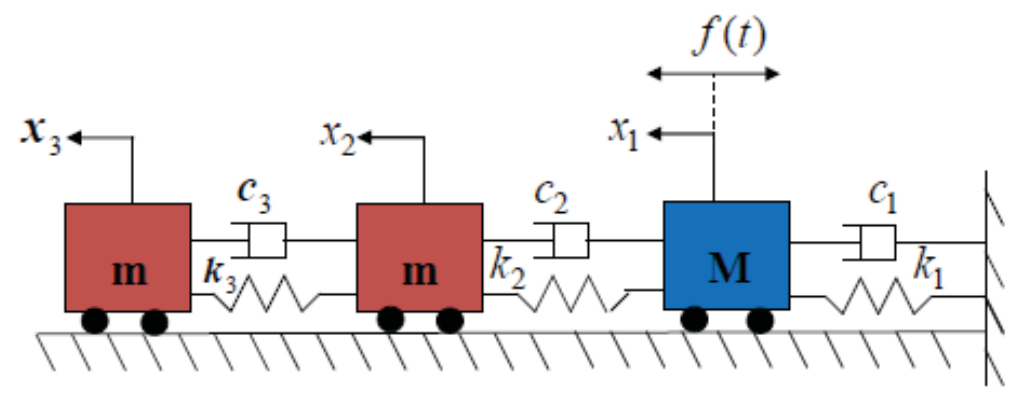

Figure 6. Single degree-of-freedom constrained tuned mass damper 
We can derive the equation of motion for the tuned mass damper problem shown in Figure 6, and it is given by

$\left[\begin{array}{ccc}M & 0 & 0 \\ 0 & m & 0 \\ 0 & 0 & m\end{array}\right]\left[\begin{array}{c}\ddot{x}_{1} \\ \ddot{x}_{2} \\ \ddot{e}\end{array}\right]+\left[\begin{array}{ccc}c_{1}+c_{2} & -c_{2} & 0 \\ -c_{2} & c_{2} & -c_{3} \\ c_{2} & -c_{2} & 2 c_{3}\end{array}\right]\left[\begin{array}{c}\dot{x}_{1} \\ \dot{x}_{2} \\ \dot{e}\end{array}\right]+\left[\begin{array}{ccc}k_{1}+k_{2} & -k_{2} & 0 \\ -k_{2} & k_{2} & -k_{3} \\ k_{2} & -k_{2} & 2 k_{3}\end{array}\right]\left[\begin{array}{c}x_{1} \\ x_{2} \\ e\end{array}\right]=\left[\begin{array}{c}1 \\ 0 \\ 0\end{array}\right] f(t)$,

where $e=x_{3}-x_{2}$.

For purpose of illustration and simulation, we assume that $M=100, c_{1}=2, k_{1}=100, m=2$, $c_{2}=c_{3}=0.4, k_{2}=10, k_{3}=1.8$, and $f(t)=10 \sin (t)$. It is clear that the natural frequency of mass $M$ is $1 \mathrm{rad} / \mathrm{s}$, which also coincides with external excitation frequency. Here, we consider lightly damped system, though both $c_{2}$ and $c_{3}$, as well as $k_{2}$ and $k_{3}$, can be adjusted actively by introducing appropriate control feedback.

Figure 7 shows the displacement of mass $M$ with and without the tuned mass damper, and the suppression of vibrational motion for mass $M$ is apparent when tuned mass damper is activated. However, in this case no relative displacement constraints are imposed. Now, when relative motion between $x_{2}$ and $x_{3}$ is confined by 1 , i.e., $|e| \leq 1$, though the effectiveness of absorbing vibration motion of mass $M$ is reduced, it is still acceptable, see Figure 8. And the results can be further improved by means of active control system design. ${ }^{3}$

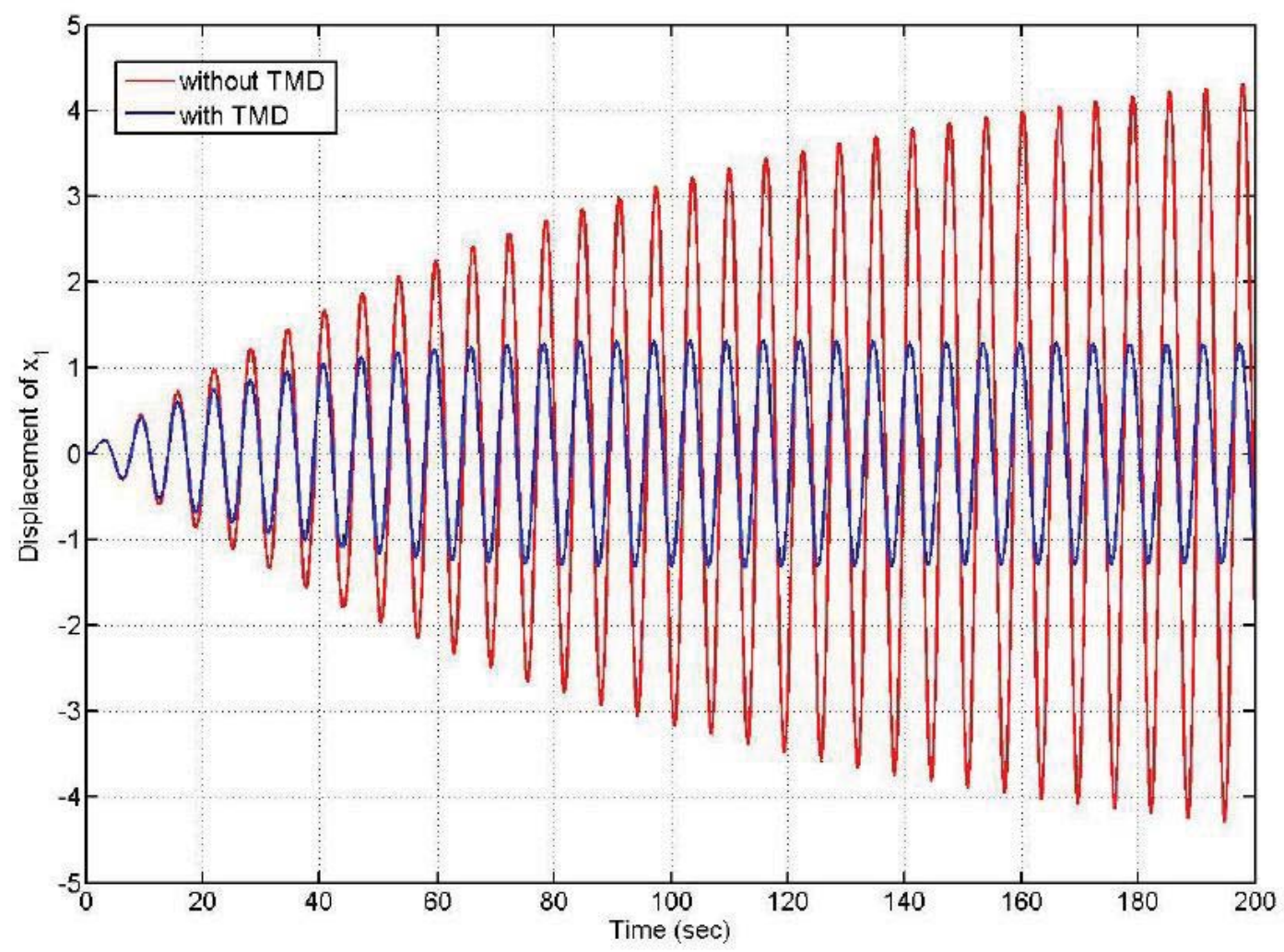

Figure 7. Displacement of mass $M$ with and without TMD 


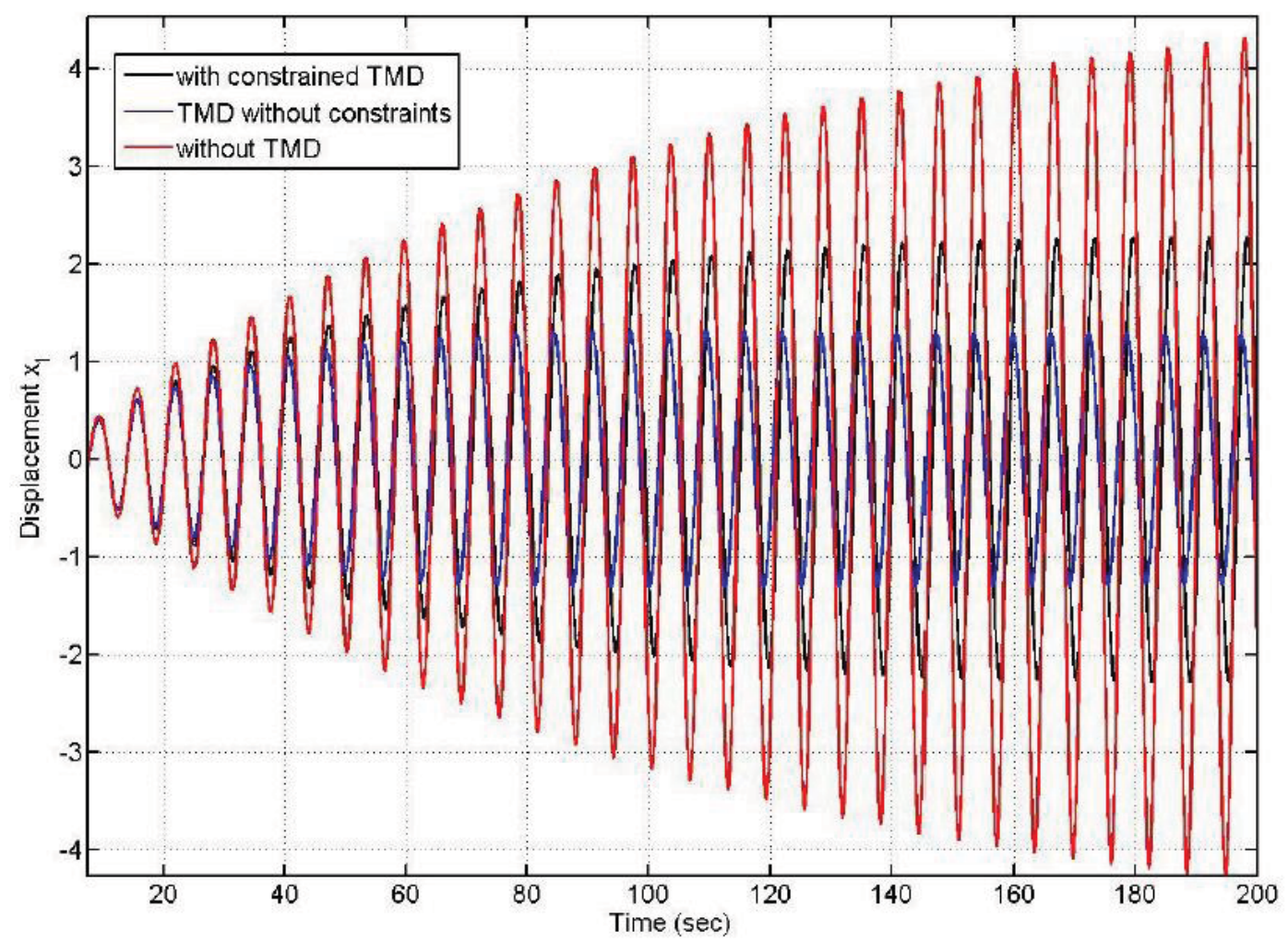

Figure 8. Displacement of mass $M$ with bounded TMD, $|e| \leq 1$.

\section{Control of Flexible Wings Using VCCTEF}

In this section, we only focus on controlling the flexible wings using VCCTEF by utilizing its model developed in Section III. To this end, recall the aeroelastic wing model from (1) and the VCCTEF dynamic model given in (3)

$$
\left\{\begin{array}{l}
\dot{x}_{e}=A_{e e} x_{e}+B_{e e} \delta_{f} \\
\ddot{\delta}_{f}+C_{f} \dot{\delta}_{f}+K_{f} \delta_{f}=b \delta_{c f}
\end{array}\right.
$$

To derive (7) we have neglected aeroelastic coupling effects. It should be noted that the dimension of $x_{e}$ depends on the number of aeroelastic modes retained in the model. Note that the first equation of (7) with bounded $\delta_{f}$ and $\dot{\delta}_{f}$ is precisely the problem setup considered in ${ }^{6}$ and. ${ }^{7}$ In this study, the actuation of $\delta_{f}$, and hence $\dot{\delta}_{f}$, is generated by the motion of VCCTEFs, and the commanded control input $\delta_{c f}$ is so designed to produce desired bounded $\delta_{f}$ and $\dot{\delta}_{f}$. The complexity of VCCTEF dynamics suggests a better management and coordination of its actuation to achieve effective wing shaping control. Therefore, the control law architecture proposed in this section consists of two integrated loops. The deflection and deflection rate saturation control law developed in ${ }^{6}$ is used as an outer loop, which is then integrated with an inner PCHbased controller ${ }^{5} 9$ for managing the VCCTEF dynamics subjected to bounds and constraints. Figure 9 shows the schematics of the proposed integrated control architecture. As shown, the control command $\delta_{c f}$ is a combination of bounded position/rate control and command shaping control. The command shaping for $\delta_{c f}$ is achieved by utilizing $\mathrm{PCH}$ approach ${ }^{5,9}$ integrated with dynamic inversion, as shown in Figure 9. 


\section{V.A. Coordinate transformation}

Given the position and rate constraints of VCCTEF, we can define a coordinate transformation to normalize VCCTEF dynamics (3). First, let $\hat{\delta}_{f}=E \delta_{f}$, where $E$ is given as

$$
E=\left[\begin{array}{ccccc}
1 & -1 & 0 & \cdots & 0 \\
0 & 1 & -1 & \cdots & 0 \\
\vdots & \vdots & \vdots & \cdots & \vdots \\
0 & 0 & 0 & \cdots & 1
\end{array}\right]
$$

then, the VCCTEF dynamics can be rewritten as follows,

$$
\ddot{\hat{\delta}}+\hat{C} \dot{\hat{\delta}}_{f}+\hat{K} \hat{\delta}_{f}=\hat{B} \delta_{c f},
$$

where $\hat{C}=E C_{f} E^{-1}, \hat{K}=E K_{f} E^{-1}$, and $\hat{B}=b E$. Note that $\hat{\delta}_{f}$ is a vector of relative deflection between two adjacent flaps, except the $m$-th flap. Alternatively, we can choose the first flap to be unconstrained. Next, we define the controlled outputs, $\bar{\delta}_{f}$ and $\dot{\bar{\delta}}_{f}$, as being the normalized deflection and deflection rate bounds by $D_{1}$ and $D_{2}$, where $D_{1}=\operatorname{diag}\left(\alpha_{1}, \alpha_{2}, \cdots, \alpha_{m}\right)$ and $D_{2}=\operatorname{diag}\left(\rho_{1}, \rho_{2}, \cdots, \rho_{m}\right)$. That is,

$$
\bar{\delta}_{f}=D_{1}^{-1} \hat{\delta}_{f} \text { and } \dot{\bar{\delta}}_{f}=D_{2}^{-1} \dot{\hat{\delta}}_{f} .
$$

Therefore, each element in $\bar{\delta}_{f}$ and $\dot{\bar{\delta}}_{f}$ is being normalized by \pm 1 . We now can rewrite Eq. (7) as follows,

$$
\left\{\begin{array}{l}
\dot{x}_{e}=A_{e e} x_{e}+B_{e e} \delta_{f} \\
\ddot{\tilde{\delta}}_{f}+\hat{C} \hat{\hat{\delta}}_{f}+\hat{K} \hat{\delta}_{f}=\hat{B} \delta_{c f} \\
\delta_{f}=E^{-1} D_{1} \bar{\delta}_{f} \\
\dot{\delta}_{f}=E^{-1} D_{2} \dot{\bar{\delta}}_{f}
\end{array}\right.
$$

\section{V.B. Bounded position/rate control design}

In this section, we'll use the extended High Performance Bounded (eHPB) control algorithm proposed by ${ }^{6}$ to design a bounded position/rate controller for suppressing the vibrational motion of the flexible wing. The construction of control law is based on the standard LQR approach for augmented control system. Consider the aeroelastic wing model; the first equation of (8). The augmented aeroelastic wing model is derived by augmenting the state $x_{e}$ with the control input $\delta_{f}$, i.e.

$$
\dot{X}=\mathcal{A} X+\mathcal{B} \dot{\delta}_{f}
$$

where

$$
X=\left[\begin{array}{c}
x_{e} \\
\delta_{f}
\end{array}\right], \mathcal{A}=\left[\begin{array}{cc}
A_{e e} & B_{e e} \\
0 & 0
\end{array}\right], \mathcal{B}=\left[\begin{array}{c}
0 \\
I_{m}
\end{array}\right]
$$

and $I_{m}$ denotes an $m \times m$ identity matrix. As shown in Section V.A, both $\delta_{f}$ and $\dot{\delta}_{f}$ can be normalized by a "state" and "input" transformation. Let $\bar{X}=T X$, where $T$ is defined by

$$
T=\left[\begin{array}{cc}
I_{n} & 0 \\
0 & D_{1}^{-1} E
\end{array}\right]
$$

and $\dot{\delta}_{f}=E^{-1} D_{2} \dot{\bar{\delta}}_{f}$. Then, (9) can be equivalently described by

$$
\dot{\bar{X}}=\overline{\mathcal{A}} \bar{X}+\overline{\mathcal{B}} \dot{\delta}_{f}
$$

where $\overline{\mathcal{A}}=T \mathcal{A} T^{-1}, \overline{\mathcal{B}}=T \mathcal{B} E^{-1} D_{2}$, and each element in $\bar{\delta}_{f}$ and $\dot{\bar{\delta}}_{f}$ is bounded by \pm 1 . The problem described in (10) is precisely the eHPB control problem considered in, ${ }^{6}$ and the following theorem provides the stabilizing control law. 
Theorem $1{ }^{6}$ Consider (10). Let $Q^{t}=Q=\operatorname{diag}\left(Q_{1}, r I_{m}\right) \geq 0$ be chosen such that $(\overline{\mathcal{A}}, Q)$ is observable; where $r>0$ is a scalar. Then, the control law

$$
\dot{\bar{\delta}}_{f}=-\frac{1}{r} \overline{\mathcal{B}}^{t} P(r) \bar{X}
$$

asymptotically stabilizes (10) subject to the normalized constraints, where, for a given $r>0, P(r)$ is the unique stabilizing solution to the following algebraic Riccati equation

$$
\overline{\mathcal{A}}^{t} P(r)+P(r) \overline{\mathcal{A}}-\frac{1}{r} P(r) \overline{\mathcal{B}} \overline{\mathcal{B}}^{t} P(r)+Q=0,
$$

and with $r(\bar{X})=\min \{r \mid \bar{X} \in \mathcal{E}(r), \forall t \geq 0\} ;$ where

$$
\begin{aligned}
& \mathcal{E}(r)=\left\{\bar{X} \mid \bar{X}^{t} P(r) \bar{X} \leq \beta\right\}, \\
& \beta=\left[\max \left(\operatorname{diag}\left(\overline{\mathcal{B}}^{t} P \overline{\mathcal{B}} / r^{2}\right)\right)\right]^{-1}
\end{aligned}
$$

In summary, the control law given in (11) guarantees that the augmented closed-loop "state" trajectories started within the Lyapunov ellipsoid $\mathcal{E}(r)$ will asymptotically converge to the origin. Though the input to the aeroelastic wing model is $\bar{\delta}_{f}$, the controller derived in (11) is a dynamic one, since $\bar{X}$ consists of both $x_{e}$ and $\bar{\delta}_{f}$. Hence, we can describe $\dot{\bar{\delta}}_{f}$ more explicitly as

$$
\dot{\bar{\delta}}_{f}=-K_{1}(r) x_{e}-K_{2}(r) \bar{\delta}_{f}
$$

where $K_{1}(r)$ and $K_{2}(r)$ can be extracted from (11). It is important to note that the control actuation; be it $\bar{\delta}_{f}$ or $\dot{\bar{\delta}}_{f}$, can not be generated instantaneously. In the case of VCCTEF, the control actuation needs to go through a negotiated process in order to generate a smooth flap profile for flutter suppression.

\section{V.C. PCH-based VCCTEF actuation}

As indicated earlier, the constrained VCCTEF dynamics in application to the flexible wing control poses a great challenge. Given the bounded control law (11), this section is to design an effective VCCTEF control management scheme to achieve desired flap profile. In this regard, the PCH-based control approach ${ }^{5,9}$ is proposed, which utilizes the dynamic inversion scheme. Relatively speaking, the actuator dynamics including the elastomer can be modeled more accurately and its dynamics inherently stable, so we should not expect large mismatch from dynamics inversion process. Therefore, we consider PCH-base approach a suitable scheme, and it is used as an integrated part of command shaping for outer loop input command model.

To derive the dynamic inversion for VCCTEF model, recall

$$
\ddot{\hat{\delta}}+\hat{C} \dot{\hat{\delta}}_{f}+\hat{K} \hat{\delta}_{f}=\hat{B} \delta_{c f} \text {. }
$$

Note that $\hat{B}$ is an invertible matrix. Since both $\dot{\hat{\delta}}_{f}$ and $\hat{\delta}_{f}$ are measurable, we can define

$$
\delta_{c f}=-\hat{B}^{-1}\left(\hat{C} \dot{\hat{\delta}}_{f}+\hat{K} \hat{\delta}_{f}-v\right)
$$

where $v$ is a new control input. Substituting the above into (13) yields

$$
\ddot{\hat{\delta}}_{f}=v,
$$

and we can choose $v$ to be a simple linear position and rate controller. Because the VCCTEF motions are bounded and constrained, the commanded flap deflection angle $\delta_{c f}$ will differ from the actual actuator flap deflection angle $\delta_{f}$. In this case, the PCH implementation will help modify the commanded model so that $\delta_{f}$ follows closely with $\delta_{c f}$. The dynamic inversion based PCH scheme for VCCTEF model is shown in Figure 9.

The overview of the proposed control scheme is as follows. As noted earlier, if we discount the VCCTEF actuator dynamics, the bounded controller given in (11) guarantees the closed-loop stability, provided that 
the initial conditions originate from a bounded region. As shown in Figure 9, the control law (11) serves as a "reference" generator and outputs the reference commands. The $\mathrm{PCH}$ signal $\hat{v}$ is defined as

$$
\hat{v}=v-v_{a}
$$

and it is fed back as a compensation to shape the reference model. That is,

$$
\dot{\bar{\delta}}_{f}=-K_{1}(r) x_{e}-K_{2}(r) \bar{\delta}_{f}-\hat{K}_{3} \hat{v},
$$

where $\hat{K}_{3}=k_{3} D_{2}$ and $k_{3}>0$ is a design parameter. Therefore, the commanded flap deflection and deflection rate are given by

$$
\begin{aligned}
& \left(\hat{\delta}_{f}\right)_{\mathrm{cmd}}=\hat{K}_{1} \bar{\delta}_{f} \\
& \left(\dot{\hat{\delta}}_{f}\right)_{\mathrm{cmd}}=\hat{K}_{2} \dot{\bar{\delta}}_{f}
\end{aligned}
$$

where $\hat{K}_{1}=k_{1} \hat{K} D_{1}$ and $\hat{K}_{2}=k_{2} \hat{C} D_{2}$, and $k_{1}>0, k_{2}>0$ are the design parameters.

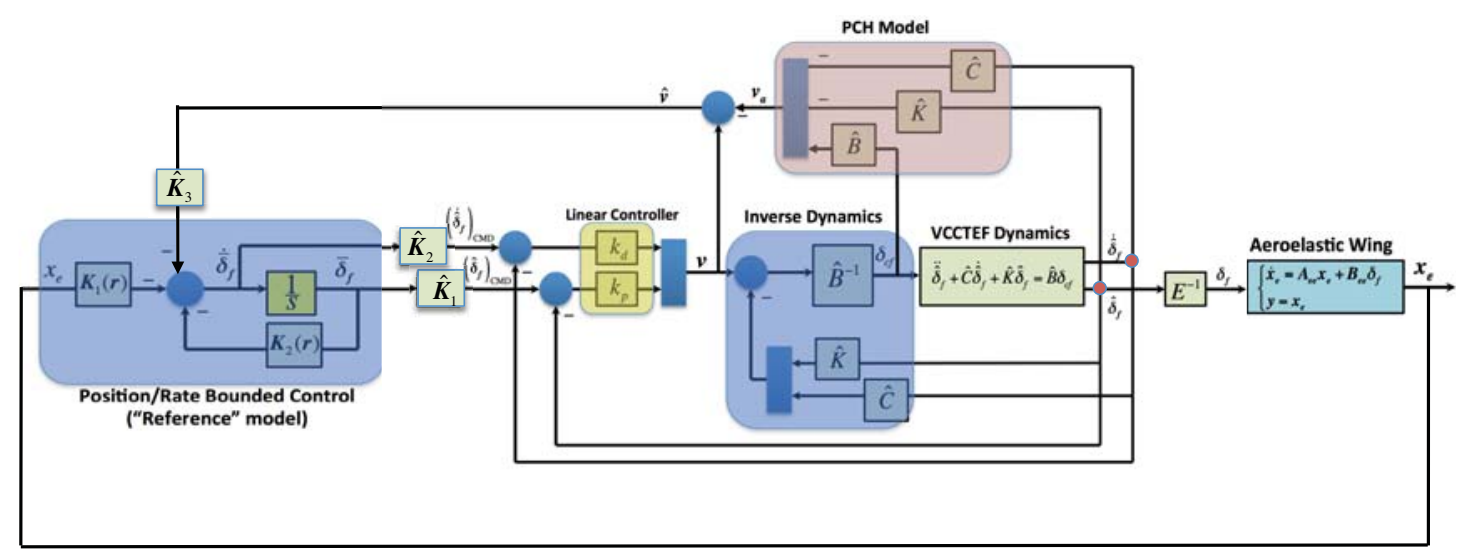

Figure 9. Dynamic inversion based PCH scheme for the control of VCCTEFs

\section{Simulation Results}

Here, we present an application of proposed PCH-based bounded control law to the GTM aeroelastic wing as an illustrative example. The GTM is configured with 15 VCCTE flaps in each wing; hence, $m=15$, and it is considered at cruise condition at Mach 0.92 and altitude of 35,000 ft. At this condition, the GTM has already exhibited fluttering behavior. The relative deflection angle and deflection rate between the two adjacent flaps are, respectively, $2 \mathrm{deg}$ and $60 \mathrm{deg} / \mathrm{sec}$. That is, $\left|\theta_{i-1}-\theta_{i}\right| \leq 2^{\circ},\left|\theta_{15}\right| \leq 2^{\circ}$, and $\left|\dot{\theta}_{i-1}-\dot{\theta}_{i}\right| \leq 60 \mathrm{deg} / \mathrm{sec}$ and $\left|\dot{\theta}_{15}\right| \leq 60 \mathrm{deg} / \mathrm{sec}$. In addition, we consider the flap actuator dynamics at two natural frequencies; $10 \mathrm{~Hz}$ ad $30 \mathrm{~Hz}$, and damping ratio of 0.8 . The elastomer has the natural frequency of $10 \mathrm{~Hz}$ and damping ratio of 0.8 . In this study, we have chosen the design parameters $k_{1}, k_{2}$, and $k_{3}$ to be: $k_{1}=k_{2}=0.5$ and $k_{3}=0.1$.

For better illustration, we only consider five aeroelastic modes in this study; including the two unstable (anti-symmetric) wing bending modes. The objective of bounded control law design then is to suppress the vibrational motion of the wings using VCCTEFs. The consideration of meeting, in addition, the overall aircraft handling qualities/performance specifications is subject of future research. Table 1 contains the list of aeroelastic modal frequency and damping considered in the simulation.

The proposed control law architecture is able to asymptotically stabilize the unstable modes when the VCCTEF actuator frequency is at either $10 \mathrm{~Hz}$ or $30 \mathrm{~Hz}$. In the case of $10 \mathrm{~Hz}$, Figures 10 and 11 show that the relative flap actuation are all bounded by \pm 1 . Moreover, Figures 12 and 13 show the actual individual flap motion. Figure 14 shows the closed-loop response in the modal coordinates. In generating the above results we have to pay special attention to the choice of initial conditions, for the limited/bounded control 
Table 1. Aeroelastic modal data for GTM

\begin{tabular}{|c|c|c|}
\hline Mode ID & Frequency (rad/sec) & Damping \\
\hline \hline unstable & 18.1 & -0.0025 \\
\hline unstable & 37.6 & -0.0283 \\
\hline bending & 20.3 & 0.135 \\
\hline bending & 42.2 & 0.194 \\
\hline torsion & 69.1 & 0.0092 \\
\hline
\end{tabular}

authority will not render global closed-loop stability. In this regard, Figure 15 indicates the trade between the control effort, in terms of $r$, and achievable ellipsoid, in terms of $\left\|x_{e}\right\|$. As can be seen that increasing the control effort; by reducing $r$, reduces the size of Lyapunov ellipsoid. In other words, large control effort can be implemented when closer to the "origin," and small gain is implemented when far away from the origin.

One important note worth mentioning is that when we follow the conventional approach, that is, the bounded control law (11) is applied directly to drive the VCCTEF dynamics which in turn affects the flexible wing, the control law fails to stabilize the closed-loop system when the VCCTEF actuator bandwidth is lower, say at $10 \mathrm{~Hz}$. Therefore, the advantage of PCH based control modification is the adaptation capability of the control system for varying actuator dynamics.

\section{Conclusion}

In this paper, we presented a PCH-based control system design approach that utilized the VCCTEF model inversion integrated with the bounded input and input rate control law. Because of the complexity of the VCCTEF dynamics, the PCH was applied to act as a control modifier to shape the command reference that is achievable, given the constraints on relative flap deflection and deflection rate. A simple TMD model was used to demonstrate that the concept of VCCTEF is efficient in suppressing the vibrational motion, and the proposed control architecture can be considered, in an extent, as an active tuned-mass-damper for flexible aircraft systems. It was also shown that when the bandwidth of flap actuator dynamics was low, the conventional control approach failed to stabilize the closed-loop system, while the proposed control architecture was able to. On the other hand, an alternative control approach might be to take the actuator dynamics into account as part of plant model, and design the bounded state/input control for the augmented system. This is a subject of ongoing research.

\section{Acknowledgments}

The authors like to thank Dr. Thomas Lombaerts of DLR for many insightful discussions on PCH modeling. 


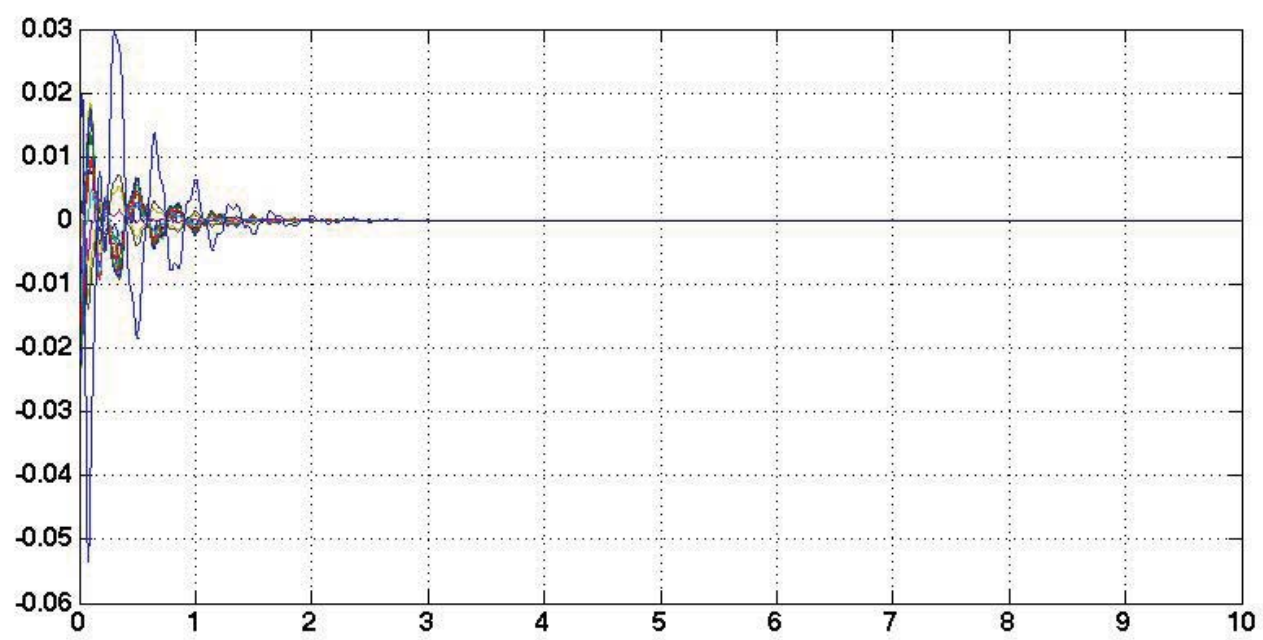

Figure 10. Normalized relative flap deflection angle $\bar{\delta}_{f}$ time history.

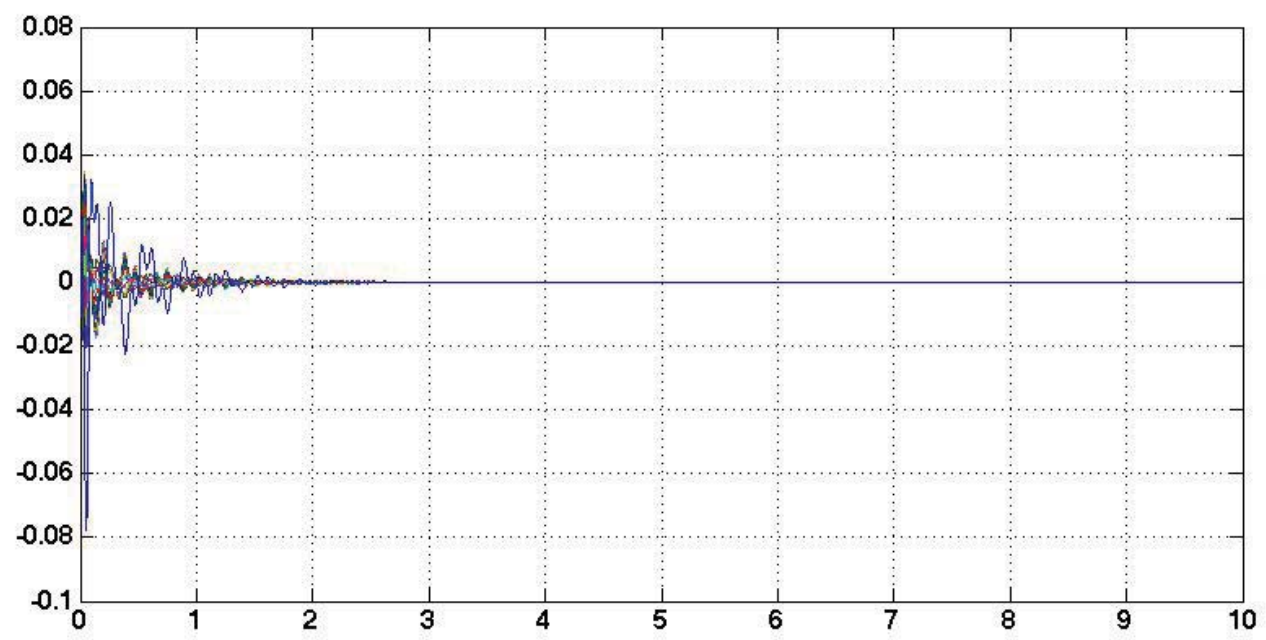

Figure 11. Normalized relative flap deflection rate $\dot{\bar{\delta}}_{f}$ time history. 


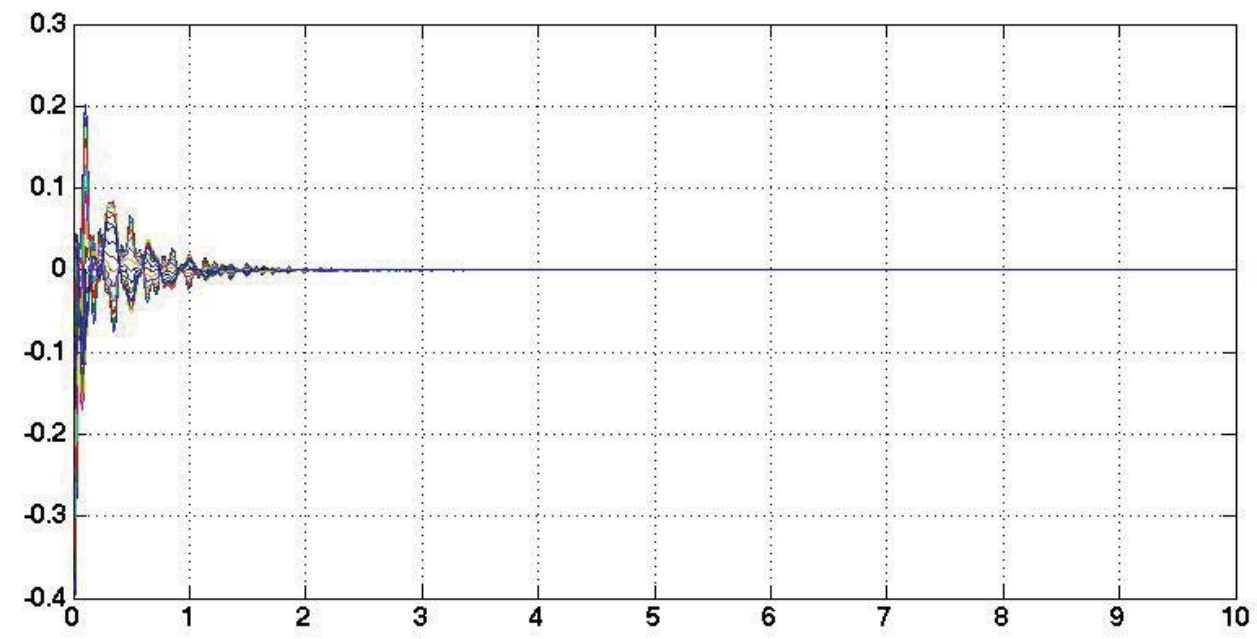

Figure 12. Individual flap deflection angle $\delta_{f}$ (deg) time history.

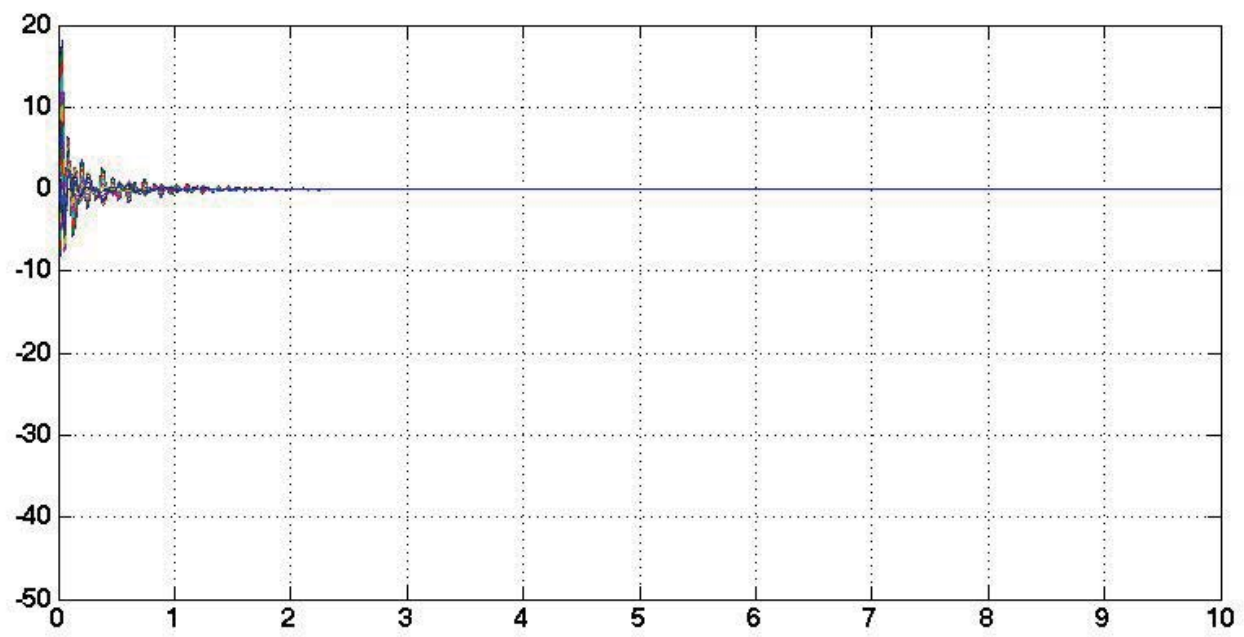

Figure 13. Individual flap deflection rate $\dot{\delta}_{f}(\mathrm{deg} / \mathrm{sec})$ time history. 


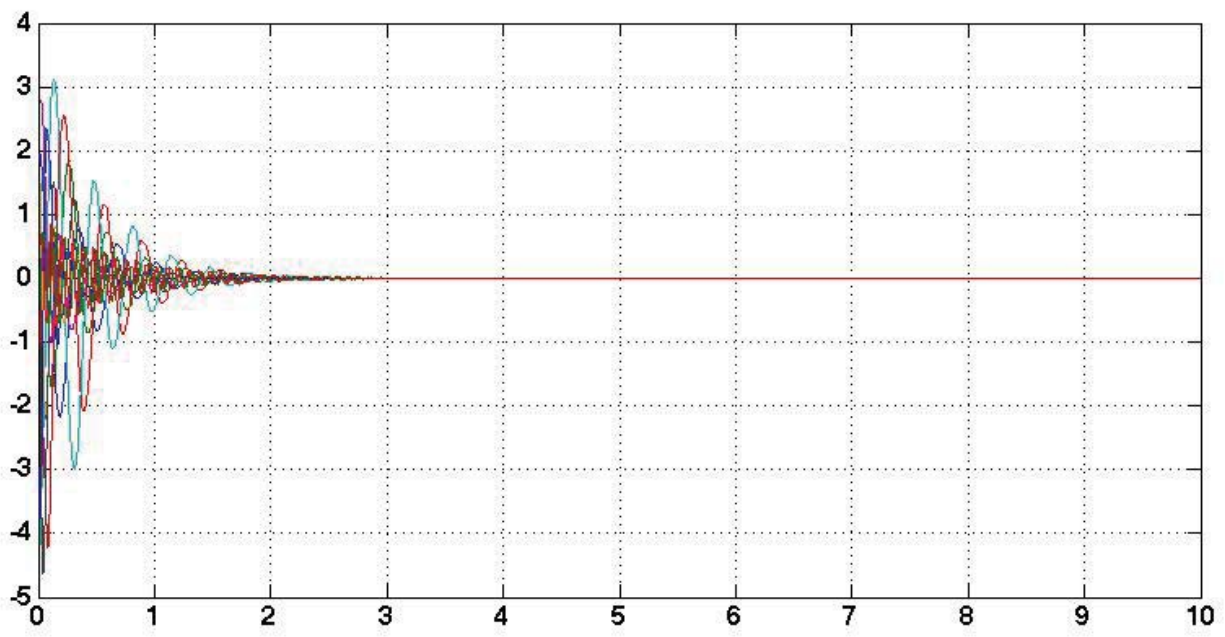

Figure 14. Closed-loop response in modal coordinates subject to initial conditions.

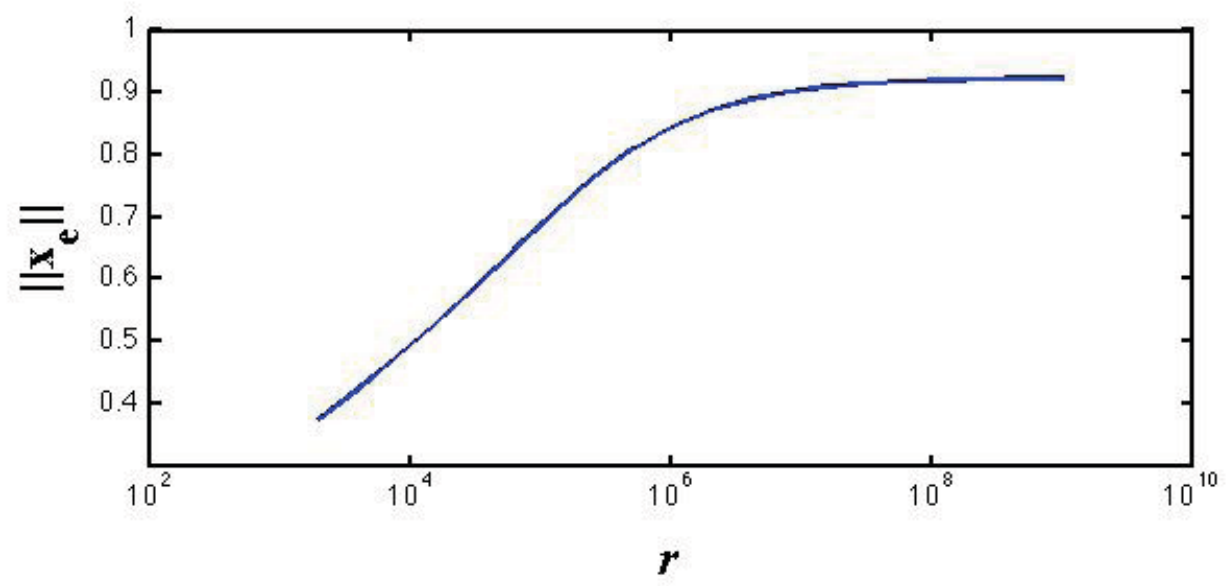

Figure 15. Achievable ellipsoid as function of control effort $(r)$. 


\section{References}

${ }^{1}$ Urnes, J., Nguyen, N., Ippolito, C., Totah, J., Trinh, K., Ting, E., " 'A Mission-Adaptive Variable Camber Flap Control System to Optimize High Lift and Cruise Lif-to-Drag Ratios of Future N+3 Transport Aircraft," AIAA Aerospace Sciences Meeting, Grapevine, TX, 2013.

${ }^{2}$ Rao, S.S., Mechanical vibrations, 3rd edition, Addison Wesley, 1995.

${ }^{3}$ Chang, C.C., Yang, H.T.Y., Swei, S.M., "'Some Techniques in Optimal Control of Building Frames," Structural Dynamic Systems Computational Techniques and Optimization: Techniques in Buildings and Bridges, edited by C.T. Leondes, Gordon and Breach Science Publishers, 1999.

${ }^{4}$ Swei, S., Zhu, G., Nguyen, N., "Integrated Model Reduction and Control of Aircraft with Flexible Wings,"' AIAA Guidance, Navigation, and Control Conference, Boston, MA, 2013.

${ }^{5}$ Lombaerts, T.J.J., Looye, G.H.N., Chu, Q.P., Mulder, J.A., "'Pseudo Control Hedging and its Application for Safe Flight Envelope Protection," AIAA Guidance, Navigation, and Control Conference, Toronto, Ontario, Canada, 2010.

${ }^{6}$ Shewchun, J.M., Feron, E., "'High Performance Bounded Control of Systems Subject to Input and Input Rate Constraints,"' AIAA Guidance, Navigation, and Control Conference, New Orleans, LA, 1997.

${ }^{7}$ Hess, R.A., Snell, S.A., "'Flight Control System Design with Rate Saturating Actuators,"' Journal of Guidance, Control, and Dynamics, Vol. 20, No. 1, pp. 90-96, 1997.

${ }^{8}$ Lin, Z., "Semi-global Stabilization of Linear Systems with Position and Rate-limited Actuators,"' Systems 83 Control Letters, 30, pp. 1-11, 1997.

${ }^{9}$ Johnson, E.N., Calise, A.J., "'Pseudo-Control Hedging: A New Method for Adaptive Control,"' Advances in Navigation Guidance and Control Technology Workshop, 2000. 